\title{
Chronic Exposure to External Low-Dose Gamma Radiation Induces an Increase in Anti-inflammatory and Anti-oxidative Parameters Resulting in Atherosclerotic Plaque Size Reduction in $\mathrm{ApoE}^{--}$Mice
}

\author{
T. G. Ebrahimian, ${ }^{a, 1}$ L. Beugnies, ${ }^{a}$ J. Surette, ${ }^{b}$ N. Priest,${ }^{b}$ Y. Gueguen, ${ }^{a}$ C. Gloaguen, ${ }^{a}$ M. Benderitter,${ }^{a}$ J. R. \\ Jourdain ${ }^{a}$ and $\mathrm{K}$. Tack $^{a}$ \\ ${ }^{a}$ Institut de Radioprotection et de Sureté Nucléaire (IRSN), PRP-HOM, SRBE, Fontenay-aux-Roses, France; and ${ }^{b}$ Radiobiology and Health, \\ Canadian Nuclear Laboratories, Chalk-River, Ontario KOJ 1J0, Canada
}

Ebrahimian, T. G., Beugnies, L., Surette, J., Priest, N., Gueguen, Y., Gloaguen, C., Benderitter, M., Jourdain, J. R. and Tack, K. Chronic Exposure to External Low-Dose Gamma Radiation Induces an Increase in Anti-inflammatory and Anti-oxidative Parameters Resulting in Atherosclerotic Plaque Size Reduction in $\mathrm{ApoE}^{-/}$Mice. Radiat. Res. 189, 187196 (2018).

Populations living in radiation-contaminated territories, such as Chernobyl and Fukushima, are chronically exposed to external gamma radiation and internal radionuclide contamination due to the large amount of ${ }^{137} \mathrm{Cs}$ released in the environment. The effect of chronic low-dose exposure on the development of cardiovascular diseases remains unclear. Previously reported studies have shown that low-dose radiation exposure could lead to discrepancies according to dose rate. In this study, we examined the effect of very low-dose and dose-rate chronic external exposure on atherosclerosis development. $A \mathbf{p o E}^{-1-}$ mice were chronically irradiated with a gamma source for 8 months at two different dose rates, 12 and $28 \mu \mathrm{Gy} / \mathrm{h}$, equivalent to dose rates measured in contaminated territories, with a cumulative dose of 67 and $157 \mathrm{mGy}$, respectively. We evaluated plaque size and phenotype, inflammatory profile and oxidative stress status. The results of this study showed a decrease in plaque sizes and an increase in collagen content in $\mathrm{ApoE}^{-/-}$mice exposed to $28 \mu \mathrm{Gy} / \mathrm{h}$ for 8 months compared to nonexposed animals. The plaque phenotype was associated with an increase in anti-inflammatory and antioxidative gene expression. These results suggest that chronic low-dose gamma irradiation induces an upregulation of organism defenses leading to a decrease in inflammation and plaque size. To our knowledge, this is the first study to describe the possible effect of chronic external very low-dose ionizing radiation exposure for 8 months. This work could help to identify the potential existence of a dose threshold, below that which harmful effects are not exhibited and

Editor's note. The online version of this article (DOI: 10.1667/ RR14823.1) contains supplementary information that is available to all authorized users.

1 Address for correspondence: Institut de Radioprotection et de Sureté Nucléaire (IRSN), PRP-HOM, SRBE, Fontenay-aux-Roses, France; email: teni.ebrahimian@irsn.fr. beneficial effects are potentially observed. Furthermore, these findings permit consideration of the importance of dose rate in radiation protection. (c) 2018 by Radiation Research Society

\section{INTRODUC'TION}

Epidemiological studies of Japanese atomic bomb survivors $(1,2)$ and medically exposed radiotherapy patients (3) indicate long-term risks of non-cancer health effects, such as cerebrovascular and, even more commonly, cardiovascular diseases (CVDs) after exposure to ionizing radiation at moderate doses. The occurrence of cardiovascular morbidity and mortality in the context of radiationinduced heart diseases usually occurs over a period of several years $(4,5)$. There is emerging evidence of excess risk of CVDs resulting from exposure to doses well below those previously considered safe, which occur late after radiation exposure in various occupationally-exposed groups (6). However, it remains unclear whether low-dose exposure poses long-term degenerative risks specifically for CVD.

The concept that exposure of the heart and the vasculature to high doses of ionizing radiation can cause CVD was first recognized in the late 1960s (7). This was mainly related to the clinical observation of cardiovascular complications in radiation-treated survivors of Hodgkin's lymphoma and other childhood cancers. Later, larger scale epidemiological studies established a clear association between therapeutic doses for thoracic radiotherapy and an increased risk of CVD in long-term cancer survivors, particularly patients treated for breast cancer, confirming the earlier observations (8).

While the mechanism by which radiation causes heart disease remains unclear, it is known that radiation, at least partially, causes or promotes atherosclerosis. In addition to epidemiological studies, animal models can be useful to 
investigate radiation-induced cardiovascular disorders. However, the interaction of radiation exposure with other atherogenic risk factors is difficult to study in these animal models. The effect of ionizing radiation on the development and progression of atherosclerosis has been investigated in various animal models, which have been reviewed. For example, Stewart et al. examined the development and progression of atherosclerotic lesion in apolipoprotein $\mathrm{E}^{-{ }^{-i}}$ (ApoE ${ }^{--}$) mice after single-dose irradiation $(14 \mathrm{~Gy}$ ) of the neck region (9). There was no major increase in the total plaque burden in the exposed carotid arteries, although plaque quality was changed, acquiring inflammatory characteristics. Indeed, the plaques showed a macrophagerich core, low-collagen content and intra-plaque hemorrhage, which are known to render human atherosclerotic plaque unstable and prone to rupture.

Atherosclerosis is a multifactorial disease, resulting from interactions between genetic and environmental factors that radiation exposure might modify. Inflammatory processes are manifested by enhanced biosynthesis of mediators of inflammation. The mediators and reactions include interleukin-6, monocyte chemoattractant protein-1 (MCP-1), intercellular adhesion molecule-1 (ICAM-1), endothelialselectin, adhesion/infiltration of monocytes, oxidation of low-density lipoprotein (LDL) and production of foam cells (10). All of these factors contribute to plaque progression (11). For radiation doses above 1 to $2 \mathrm{~Sv}$, in vitro and in vivo studies have shown that several mechanisms may play a relevant role in radiation-induced cardiovascular effects (12-15). These effects include endothelial dysfunction, inflammation, oxidative stress, alterations of coagulation and platelet activity, DNA damage, senescence and cell death. Data from experimental models of high-dose radiation are generally consistent with at least some of the human data on high-dose radiation, which include accelerating the development of atherosclerosis and predisposing to the formation of an inflammatory, thrombotic plaque phenotype, especially in animals that are genetically predisposed to this disease $(9,16-18)$. The findings demonstrate that both total dose and dose rate have implications for CVDs.

In general, low-dose radiation delivered at a low dose rate $\left(1 \mathrm{mGy} \cdot \mathrm{min}^{-1}\right)$ during either early- or late-stage disease was protective, slowing the progression of the disease $(19,20)$. Conversely, high-dose-rate (about $150 \mathrm{mGy} \cdot \mathrm{min}^{-1}$ ) exposure during early-stage disease induced both protective and detrimental effects, suggesting that multiple mechanisms may influence the outcome. Moreover, a recently reported study from our laboratory has shown that chronic internal low-dose ${ }^{137} \mathrm{Cs}$ exposure for 9 months enhances atherosclerotic lesion stability by inhibiting pro-inflammatory cytokine and MMP production, resulting in collagen-rich plaque with less macrophage content (21). Similarly, it has been reported that in diabetic mice, repeated low-dose exposure reduced lipid levels, attenuated inflammation and reduced cardiac expression of multiple inflammatory agents (22).
Likewise, multiple low-dose irradiation of mice reduced inflammatory signaling in a mouse model of asthma (23). These results suggest that low doses of radiation have an effect that is opposite of that from high doses in atherosclerosis development, and that dose rate is a very important parameter to consider. Dose rate dependent of inflammatory response and endothelial cell function has been demonstrated recently in published in vitro experiments (24).

Since anti-inflammatory effects of low-dose radiation were reported in models of chronic inflammatory diseases, we therefore hypothesized that chronic external low-dose exposure would regulate atherosclerosis progression in mice. Genetically predisposed $\mathrm{ApoE}^{-1}$ mice were chronically exposure to 12 or $28 \mu \mathrm{Gy} \cdot \mathrm{h}^{-1}$ for 8 months, the cumulative dose was 67 and $157 \mathrm{mGy}$ after 8 months of exposure, respectively. We investigated atherosclerosis progression along with oxidative stress balance, inflammatory status and indices of plaque size and stability. This novel study reports on the possible effect on atherosclerosis of very low-dose (below $0.3 \mathrm{~Gy}$ ) chronic external ionizing radiation exposure for 8 months. These findings could help to identify the potential existence of a dose threshold, below which harmful effects are not exhibited and beneficial effects are potentially observed. In addition, these findings permit consideration of the importance of dose rate in radiation protection.

\section{MATERIALS AND METHODS}

\section{Animals}

$\mathrm{ApoE}^{-/-}$male mice (7-8 weeks old) were obtained from Charles River Laboratories, Wilmington, MA. Each group comprised 10 animals. ApoE ${ }^{-/}$mice are homozygous null for a functional ApoE gene on a $\mathrm{C} 57 \mathrm{BL} / 6 \mathrm{~J}$ background. Apolipoprotein $\mathrm{E}$ acts as the main ligand mediating removal of cholesterol-enriched chylomicron and VLDL remnants from the blood stream and plays an important role in lipoprotein metabolism. These mice develop atherosclerosis when fed with a normal low-fat diet. The morphological features of early-stage lesions in $\mathrm{ApoE}^{-/-}$mice are very similar to those found in humans (25). Animals were maintained in a specific-pathogen-free environment. All experiments and procedures were carried out in accordance with the Guide for the Care and Use of Laboratory Animals, published by the Canadian regulations for animal experiments, and approved by the local ethical committee of Canadian Nuclear Laboratories (CNL) in Chalk River (permit no: DRF-09-05).

\section{Irradiations}

Animals were separated into three groups: 1. nonirradiated; 2. irradiated at a dose rate of $12 \mu \mathrm{Gy} / \mathrm{h}$; and 3 . irradiated at a dose rate of $28 \mu \mathrm{Gy} / \mathrm{h}$ for 8 months. All irradiations were performed at the CNL Chalk River animal facility.

\section{Blood Sampling and Analysis}

Mice were terminally anesthetized by intraperitoneal injection of ketamine/xylazine (Ketamine 500 and Rompun 2\%, respectively). Blood was collected by intracardiac puncture with a EDTA syringe. Blood samples were centrifuged for $10 \mathrm{~min}$ at $800 \mathrm{~g}$ and plasma was harvested and frozen for subsequent analysis. Plasma cholesterol, low- 
TABLE 1

Evaluation of White and Red Blood Cell Count after Gamma-Ray Irradiation

\begin{tabular}{lccc}
\hline Number of cell/count & $0 \mu \mathrm{Gy} \cdot \mathrm{h}^{-1}$ & $12 \mu \mathrm{Gy} \cdot \mathrm{h}^{-1}$ & $28 \mu \mathrm{Gy} \cdot \mathrm{h}^{-1}$ \\
\hline White cells & $8.39 \mathrm{E}+09$ & $7.20 \mathrm{E}+09$ & $6.87 \mathrm{E}+09$ \\
Monocytes & $1.64 \mathrm{E}+08$ & $1.69 \mathrm{E}+08$ & $1.43 \mathrm{E}+08$ \\
Eosinophils & $1.00 \mathrm{E}+09$ & $2.40 \mathrm{E}+09$ & $4.20 \mathrm{E}+09$ \\
Lymphocytes & $5.70 \mathrm{E}+09$ & $5.30 \mathrm{E}+09$ & $4.70 \mathrm{E}+09$ \\
Neutrophils & $2.30 \mathrm{E}+09$ & $1.60 \mathrm{E}+09$ & $1.90 \mathrm{E}+09$ \\
Basophils & $2.50 \mathrm{E}+07$ & $2.10 \mathrm{E}+07$ & $2.70 \mathrm{E}+07$ \\
Red blood cells & $9.10 \mathrm{E}+12$ & $9.20 \mathrm{E}+12$ & $9.20 \mathrm{E}+12$ \\
MCV & 49.8 & 49.6 & 50.5 \\
HcT & 45.1 & 45.5 & 46.4 \\
MCH & 15.6 & 15.9 & 15.4 \\
MCHC & 31.4 & 32.3 & 30.6 \\
RDW & 10.9 & 10.8 & 11.3 \\
HB & 14.2 & 14.7 & 14.2 \\
Platelet & $3.2 \mathrm{E}+11$ & $5.2 \mathrm{E}+11$ & $3.9 \mathrm{E}+11$ \\
\hline
\end{tabular}

and high-density lipoproteins (LDL and HDL) and triglyceride levels were determined with an automated spectrophotometric system (Konelab 20, Biological Chemistry Reagents; Thermo Electron Corp., Saint-Herblain Cedex, France).

\section{Blood Cell Counts}

Blood was harvested by intracardiac puncture into EDTA-coated tubes for plasma isolation. Blood cell counts were performed using an MS-9 vet automatic counter. The remaining blood was centrifuged at $800 \mathrm{~g}$ for $10 \mathrm{~min}$ and plasma or serum was frozen for later use.

\section{Tissue Collection}

Hearts (including the aortic root) were separated from the aortas, embedded in optimal cutting temperature media (OCT; Sakura Fineteck, Alphen aan den Rijn, Netherlands) and snap frozen on a metal plate that was cooled with liquid nitrogen. Aortas were excised from the aortic arch to the femoral bifurcation and directly snap frozen on liquid nitrogen. The thoracic and abdominal aortas were separated for gene and protein expression analysis.

\section{Gene Expression}

Total RNA from thoracic aortas was extracted. Briefly, aortas were ground with a Precellys ${ }^{\circledR} 24$ (Bertin Instruments, France) using TRI Reagent Solution (Sigma-Aldrich ${ }^{\circledR}$ LLC, St. Louis, MO). After extraction, we performed a purification of RNA (RNeasy Mini Kit, QIAGEN $^{\circledR}$, Valencia, CA). RNA quality was checked by measuring the ratio of optical densities at 260 and $280 \mathrm{~nm}$. Real-time PCR (RTPCR) was used to analyze the mRNA levels of inflammatory cytokines and adhesion molecules ( $\amalg 4, \mathbb{L} 10$, $\amalg 13, \mathbb{L} 18$, TGF $\beta$, ESEL, IL6, MCP1 and VCAM1). Oxidative stress balance was evaluated by mRNA expression of pro- and anti-oxidant enzymes (CAT, GPx, SOD1, SOD2 and NRF2). Finally, the mRNA expression of collagen (col3a1) and MMP13 was evaluated. Real-time qPCR was performed with an ABI Prism ${ }^{\text {B }}$ 7900HT Sequence Detection System (Applied Biosystems, Courtaboeuf, France) using SYBR Green (Applied Biosystems). All samples were normalized to glyceraldehyde-3-phosphate dehydrogenase (GAPDH) or hypoxanthine-guanine phosphoribosyltransferase (HPRT). The $2^{-\triangle A C T}$ method was used to analyze the results (26). All RT-qPCR results are expressed as mean \pm SEM, and compared to expression levels of the nonirradiated group, which was set at 1 . Primers were designed using PrimerBLAST software (https://www.ncbi.nlm.nih.gov/tools/primer-blast/; NCBI, Bethesda, MD). Sequences of the forward and reverse primers used are listed in Table 1.
Histological and Immunohistochemical Analyses of Aortic Plaque Phenotype

Cryosections of $7-\mu \mathrm{m}$ thickness were cut from the origin of the aortic root throughout the aortic sinus, for histological and immunohistochemical analysis.

Oil red $O$ and picrosirius red staining. Sections (5-7 per animal) were stained for the oil red $O$ (Sigma-Aldrich) to evaluate the lipid content. Mean lesion area was calculated using Histolab ${ }^{\mathrm{TM}}$ software (GT Vision Ltd., Sudbury, UK) as described elsewhere $(17,18)$. Quantification of the lesions of nonirradiated mice was set as $100 \%$ relative to the total aortic sinus area. The 5-7 sections per animal were stained to evaluate plaque collagen content, using a standard picrosirius red staining (Sigma-Aldrich). Quantification of the redstained areas of nonirradiated controls was set as $100 \%$ relative to the total plaque area. All images were acquired using an Axiophot (Carl Zeiss, Jena, Germany).

Macrophages immunostaining. Macrophage content was determined by immunofluorescence using monoclonal anti-CD68 (cat. no. 1252; Abcam ${ }^{\circledR}$, Cambridge, MA). The aortic sinus cryosections were then incubated with primary antibody $(1: 100)$, rinsed and further incubated with fluorescently-labeled secondary antibodies (1:500) (Invitrogen ${ }^{\mathrm{TM}}$, Carlsbad, CA). At least five sections per animal were stained. Images were acquired using an Axiophot (Zeiss, Germany) and analyzed using Histolab software (GT Vision Ltd.). The presence of immunofluorescent staining was quantified and expressed in percentage of the total lesion area. For negative controls, sections were incubated with secondary antibody only. Nuclei were stained with DAPI (Vector ${ }^{\circledR}$ Laboratories, Burlingame, CA). All values were normalized to values in nonirradiated $\mathrm{ApoE}^{-/}$animals, set at $100 \%$.

Superoxide staining. Evaluation of reactive oxygen species (ROS) production in the entire plaque area of the aortic sinus was performed by dihydroethidium staining (DHE, $2 \mathrm{~m} M$; Molecular Probes ${ }^{\mathbb{E}}$, Eugene, OR), applied for $30 \mathrm{~min}$ at $37^{\circ} \mathrm{C}$. For negative control, sections were incubated with PBS $1 \times$ only. The slides were independently examined on a blinded basis for the level of ROS staining, using a 0 - to 4-point intensity gradient.

\section{Statistical Analysis}

Experiments were performed with 10 animals per group per experimental condition. To check for normal distribution, we performed a Shapiro-Wilk test. All data followed a Gaussian distribution. One-way ANOVA and Student's $t$ test were used to compare irradiated and nonirradiated animal results. All results are expressed as mean \pm SEM. Statistical software used was SigmaPlot ${ }^{\mathrm{TM}}$ version 11.0 (SPSS Inc., France) was used for all statistical analysis. Results with $P<0.05$ were considered statistically significant.

\section{RESULTS}

Chronic External Exposure to Low-Dose and Low-DoseRate Ionizing Radiation has No Effect on Animal General Health Parameters, Plasma Lipid Levels and White Blood Count

Plasma concentrations of total cholesterol, HDL and LDL were equivalent in all groups irrespective of the dose and dose rate (Table 1), as well as white blood counts (Table 2).

\section{Chronic Exposure to $28 \mu G y / h$ for 8 Months Decreases Plaque Size, Alters Plaque Inflammatory Profile and Increases Collagen Content}

Atherosclerotic lesion size was measured in the aortic sinus of $\mathrm{ApoE}^{--}$mice. Plaque areas were decreased in 
TABLE 2

Evaluation of Lipids and TG in the Plasma after Gamma-Ray Irradiation

\begin{tabular}{lccc}
\hline & $0 \mu G y \cdot h^{-1}$ & $12 \mu G y \cdot h^{-1}$ & $28 \mu G y \cdot h^{-1}$ \\
\hline Cholesterol $(M \mathrm{~m})$ & 10.8 & 9.8 & 11.2 \\
HDL (U/1) & 3.1 & 2.98 & 3.2 \\
LDL (U/1) & 8.1 & 7.2 & 7.32 \\
TG (U/1) & 0.82 & 0.83 & 0.92 \\
\hline
\end{tabular}

animals irradiated with $28 \mu \mathrm{Gy} \cdot \mathrm{h}^{-1}$ for 8 months compared to nonirradiated animals $(55.0 \% \pm 10 \%)$. Moreover, we investigated lesion composition in all groups. Macrophages are one of the major inflammatory cell types implicated in atherosclerosis progression and often the most abundant cells within lesions. To quantify macrophages within the plaque, we performed CD68 immunostaining. Our results show a decrease $(P<0.05)$ in $\mathrm{CD}^{2} 8^{+}$staining in lesions of $28 \mu \mathrm{Gy} / \mathrm{h}$ irradiated mice compared to nonirradiated animals (Fig. 2). Chemokine and adhesion molecule expression are key elements in the recruitment and diapedesis of monocytes in the vascular wall. Once inside the atherosclerotic lesion, most of these cells are transformed into macrophages. In keeping with the reduced macrophage content observed in the plaques of $28 \mu \mathrm{Gy} \cdot \mathrm{h}^{-1}$ irradiated mice, we found a parallel increase in the mRNA expression of many anti-inflammatory cytokines, measured by RTqPCR, in their aortas (Fig. 1). Aortic mRNA expression of pro-inflammatory cytokines were unchanged regardless of the group. However, the anti-inflammatory cytokines measured by RT-qPCR were significantly increased in the $28 \mu \mathrm{Gy} \cdot \mathrm{h}^{-1}$ irradiated animals compared to nonirradiated animals. Specifically, aortic mRNA expression of IL4, IL10, IL13 and IL18 were significantly increased $(P<$ 0.01) in $\mathrm{ApoE}^{-1}$ mice exposed for 8 months to $28 \mu \mathrm{Gy} \cdot \mathrm{h}^{-1}$ compared to nonirradiated animals (IL4: $50 \% \pm 10 \%$; IL10: $45.5 \% \pm 8.8 \%$; IL13: $43.2 \% \pm 8.6 \%$; IL18: $43.3 \%$ $\pm 7.7 \%$ ) (Fig. 1A). No changes were observed in cytokine expression with low-dose rate. Aortic mRNA expression levels of pro- and anti-inflammatory cytokines were measured in C57B16 animals exposed to 2 low dose-rates for 8 months and no difference was observed for all measured cytokine expression levels among the groups (Supplementary Fig. S1; http://dx.doi.org/10.1667/ RR14823.1.S1).

Quantification of picrosirius red revealed a significant increase in collagen within the plaques of mice exposed to $28 \mu \mathrm{Gy} \cdot \mathrm{h}^{-1}$ for 8 months $(159 \pm 20 \%)(P<0.05)$ compared to nonirradiated animals (Fig. 5). Finally, type III collagen mRNA expression was also found to be enhanced 1.5 -fold in the aortas of animals exposed to $28 \mu \mathrm{Gy} \cdot \mathrm{h}^{-1}$ for 8 months ( $P$ $<0.05$ ) compared to nonirradiated animals (Fig. 5).

\section{Exposure to $28 \mu G y / h$ for 8 Months Alters the Redox Balance}

Oxidative stress is a critical feature of atherosclerosis, contributing to endothelial dysfunction, LDL oxidation,
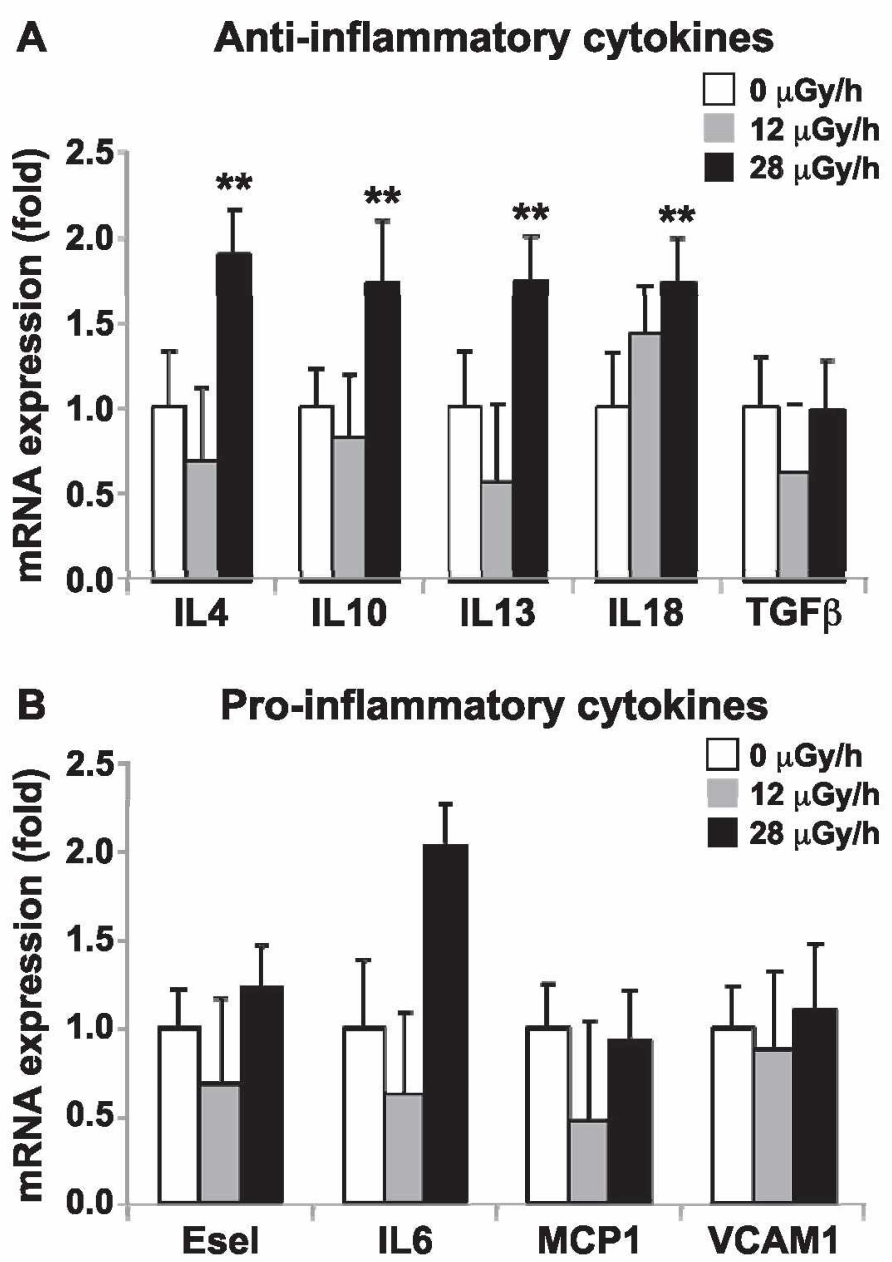

FIG. 1. Aortic mRNA expression of anti- and pro-inflammatory cytokines after 12 or $28 \mu \mathrm{Gy} / \mathrm{h}$ irradiation for 8 months. Levels of ILA, IL10, IL13, IL18, TGF $\beta$, Esel, IL6, MCP1 and VCAM1 were determined by RT-qPCR. GAPDH or HPRT were amplified and used as endogenous control. When radiation dose reached $157 \mathrm{mGy}$ (dose rate of $28 \mu \mathrm{Gy} / \mathrm{h}$ ), $\mathrm{IL} 4$, IL10, $\mathrm{LL13}$ and $\mathrm{IL} 18$ were upregulated compared to nonirradiated controls. Results are expressed as mean of fold change \pm SEM; $n=12$ animals per group.

upregulation of inflammatory pathways and MMP induction. We first evaluated plaque superoxide production by dihydroethidium (DHE) staining. No differences were noted among the animal groups at that specific time point (Fig. 3). Even at a shorter exposure of 1 month, no differences in DHE staining were observed among groups (Supplementary Fig. S2A; http://dx.doi.org/10.1667/RR14823.1.S1). Furthermore, a time course $(5,10$ and $15 \mathrm{~min})$ of DHE staining was performed on the same section and no differences were observed (Supplementary Fig. S2B). One interpretation could be that a peak was already reached within $5 \mathrm{~min}$.

However, mRNA expression levels of different antioxidant factors, such as CAT, CTAT1, SOD1 and SOD2, were significantly upregulated $(P \leq 0.05$ or $P \leq 0.01)$ in 28 $\mu \mathrm{Gy} / \mathrm{h}$ irradiated animals compared to nonirradiated animals $(27 \% \pm 0.05$-fold; $30 \% \pm 0.05$-fold; $28 \% \pm 0.04$-fold; $55 \% \pm 0.05$-fold, respectively). The same experiments were performed with C57Bl6 mice. Aortic mRNA expres- 

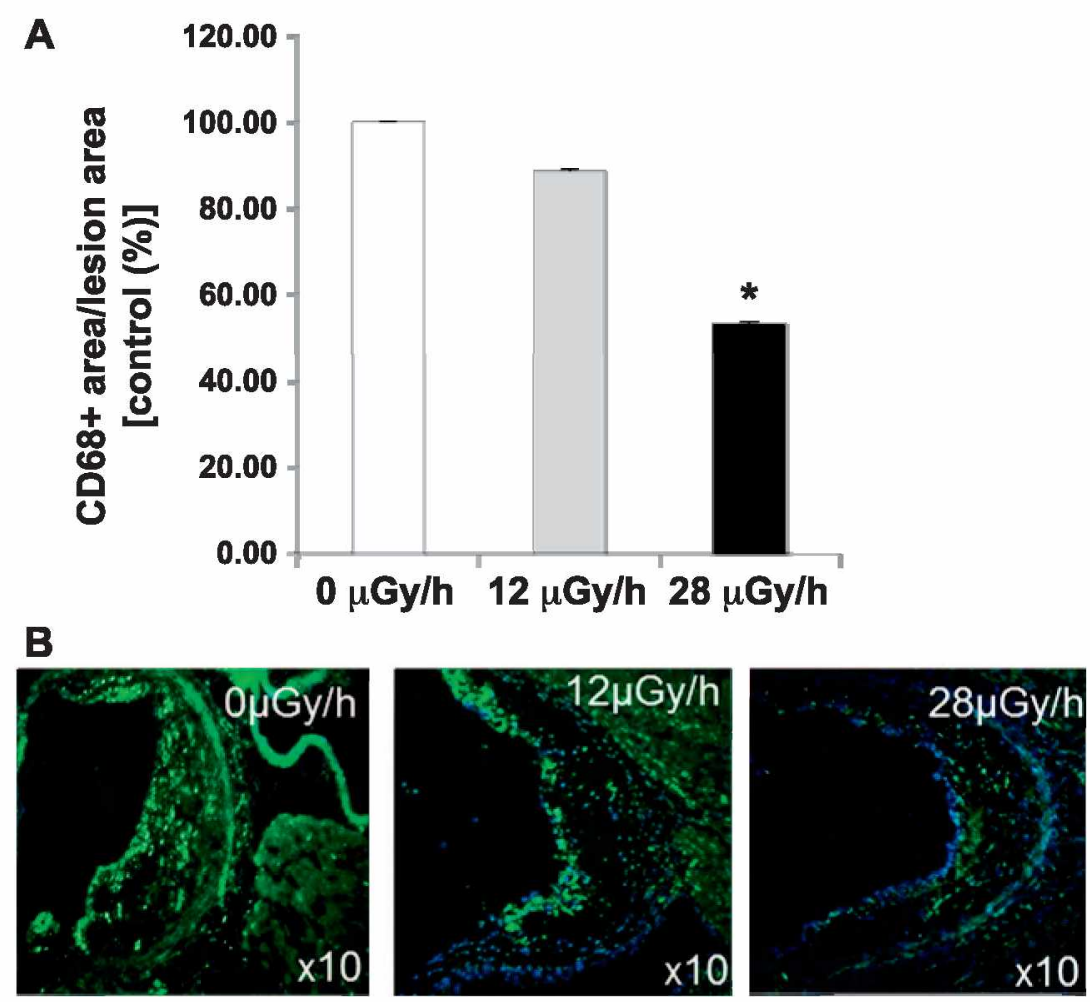

FIG. 2. Chronic gamma-ray irradiation for 8 months at $28 \mu \mathrm{Gy} / \mathrm{h}$ reduces macrophage content in atheromatous plaque. Panel A: Macrophages were detected by CD68 immunostaining (CD68 ${ }^{+}$cells: green; nuclei: blue). Representative images obtained at $100 \times$ magnification. Panel B: Quantification of CD68 ${ }^{+}$cell content. Results are expressed as mean $\pm \mathrm{SEM}$ of $\mathrm{CD} 68^{+}$surface area, proportional to nonirradiated control group (\%). $* P \leq 0.05, * * P \leq 0.01$ vs. nonirradiated group; $\mathrm{n}=5$ sections per animal.

sion levels of HO-1 and SOD1 were significantly increased in $31.25 \mu \mathrm{Gy} / \mathrm{h}$ irradiated compared to nonirradiated mice $(35 \% \pm 0.05$-fold; $20 \% \pm 0.04$-fold, respectively) (Supplementary Fig. S3; http://dx.doi.org/10.1667/ RR14823.1.S1).

\section{DISCUSSION}

The results of this study showed that chronic low-dose exposure for 8 months increased the anti-inflammatory and anti-oxidative parameters, decreased plaque size, reduced plaque macrophage content and increased collagen content. These data indicate that exposure to chronic low-dose radiation may actually promote a smaller and more stable plaque phenotype in mice associated with an increase in anti-oxidative and anti-inflammatory parameters.

In most published animal studies on radiation and atherosclerosis, exposures were for a short period of time or used higher doses. The results ranged from an increase in inflammation and atherosclerosis progression with high doses (18), to decreased atherosclerosis using very-lowdose- with low-dose-rate irradiation (19). To date, we found only three published studies that reported the effects of chronic internal gamma exposure $\left({ }^{137} \mathrm{Cs}\right)$ on the cardiovascular system, at doses comparable to post-accident exposure $(21,29,30)$. These studies yielded contradictory results. In one study, alterations in the cardiovascular systems of children who have lived in contaminated territories were reported (30), whereas the other studies showed subtle changes in blood pressure, circadian rhythm and atrial expression of some genes, or a decrease in inflammation and an increase in plaque stability $(21,29)$. One recently published study has shown that a chronic radiation dose of 0.3 Gy may lead to persistent detrimental effects on the cardiovascular system and that a high dose of 6 Gy poses high risks at both high and low dose rates (31). In vitro studies on low-dose radiation increased endothelial expression of ICAM-1 when delivered in fractionated episodes (32), while some studies have shown that low-dose radiation decreased inflammatory cytokine production, reduced migration and increased chemotaxis in macrophages, all of which could be linked to resolution of inflammation (33). There is emerging evidence that lowdose ionizing radiation modulates the function of a variety of inflammatory cells, including endothelial cells, polymorphonuclear leukocytes and macrophages $(34,35)$.

Some in vitro and in vivo studies tend to link radiation with pro-inflammatory processes. With high doses, the quality of the atheromatous plaque was different, with more inflammatory characteristics. Moreover, the presence of atypical swollen endothelial cells was observed. It is hypothesized that radiation-induced changes in endothelial function, 
A Anti-oxidant gene expression

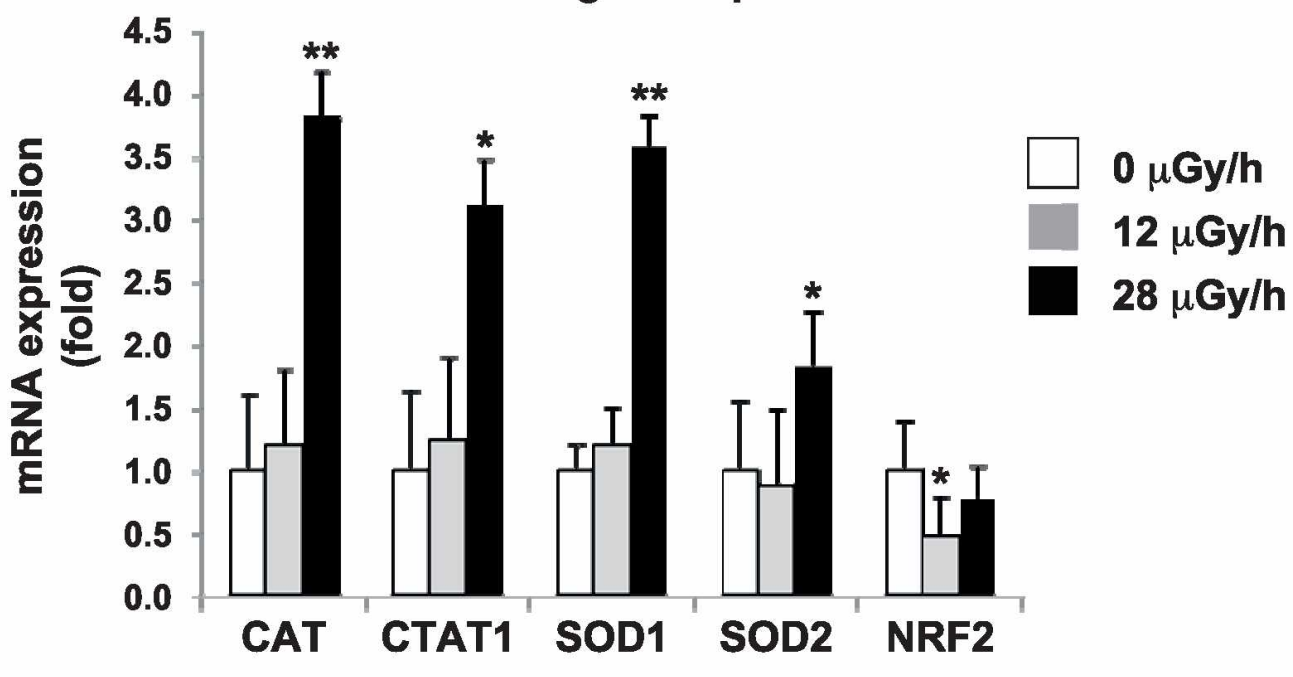

B
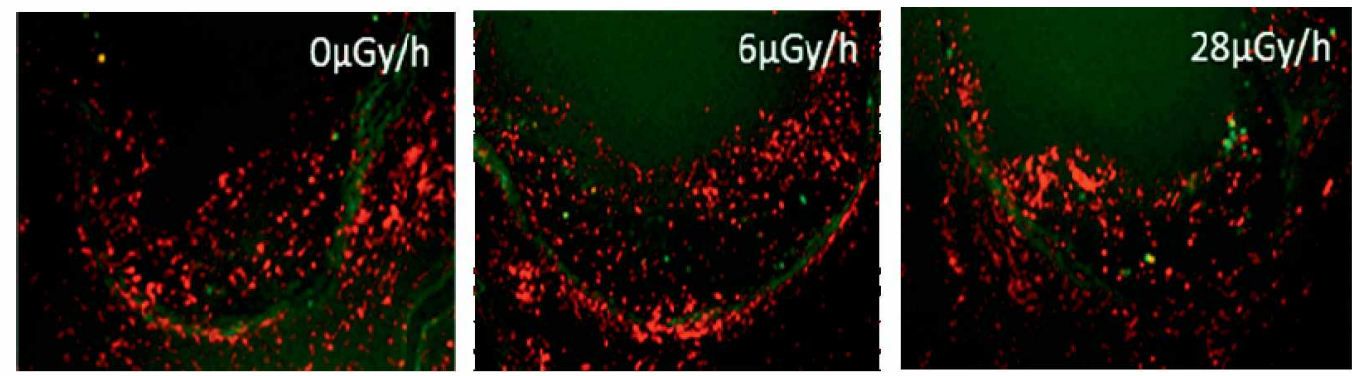

FIG. 3. Changes in oxidative stress and related enzyme expression after chronic gamma-ray irradiation for 8 months at $28 \mu \mathrm{Gy} / \mathrm{h}$. Panel A: Qualitative analyses did not reveal any differences in aortic mRNA expression of NRF2 between irradiated and nonirradiated groups, measured by RT-qPCR. However, aortic mRNA expression of CAT, CTAT1, SOD1 and SOD2 was significantly increased. $* P \leq 0.05, * * P \leq 0.01$ vs. nonirradiated group. Panel B: Representative images of superoxide production within the atheromatous plaque evaluated by DHE staining after 8 months of radiation exposure ( $200 \times$ magnification). $n=5$ sections per animal. Results are expressed as mean $\pm \mathrm{SEM} ; \mathrm{n}=12$ animals per group.

together with induced-endothelial cell death and exposure of thrombotic elements of the underlying sub-endothelium, lead to chronic inflammation and the development of vulnerable plaque (9). The effects of chronic low-dose rate compared to acute exposure were also evaluated in $\mathrm{ApoE}^{-/-}$female mice (60 days) chronically irradiated for 300 days with gamma rays at two different dose rates ( 1 and $20 \mathrm{mGy} / \mathrm{h}$ ), with total cumulated dose of 0.3 and $6 \mathrm{~Gy}$, respectively. Mice acutely exposed to 0.3 or $6 \mathrm{~Gy}$ showed increased atherogenesis compared to the age-matched controls and this effect was persistent. When the same doses were delivered at a low-dose rate over 300 days, significant effect on global development of atherosclerosis was observed again, although at $0.3 \mathrm{~Gy}$, the effects were limited to the descending thoracic aorta. Further research by the same group revealed that a more clinically relevant fractioned radiation protocol $(20 \times 2$ Gy in 4 weeks) also predisposed to the formation of an inflammatory plaque (18).

With low doses (under $300 \mathrm{mGy}$ ) and dose rates, the response warrants further investigation. Most animal studies describe a protective effect of low-dose radiation in the setting of inflammatory disease depending on the dose rate.

Our current study is in line with some of the previously reported results, and we demonstrated that chronic external low-dose $(150 \mathrm{mGy})$ irradiation at a low-dose rate (28 $\mu \mathrm{Gy} \cdot \mathrm{h}^{-1}$ ) induces a protective effect in atherosclerotic lesions. The importance of the dose, as well as the dose rate, even after chronic low-dose exposure, was confirmed in animal models (19) and in a cultured endothelial cell model $(24,36)$.

Our investigation of the effects of chronic exposure revealed that indices of anti-inflammatory parameters were actually increased in irradiated compared to nonirradiated mice when the dose reached $150 \mathrm{mGy}$ with the highest lowdose rate $\left(28 \mu \mathrm{Gy} \cdot \mathrm{h}^{-1}\right)$. Indeed, we observed an increase in $\mathrm{IL} 4, \mathrm{IL} 10$, IL13 and IL18, associated with a decrease in macrophage content within the plaque, after 8 months of exposure to $28 \mu \mathrm{Gy} \cdot \mathrm{h}^{-1}$, compared to nonirradiated animals. Our results are in accordance with some reports showing the anti-inflammatory effect of low-dose radiation $(37,38)$ and 

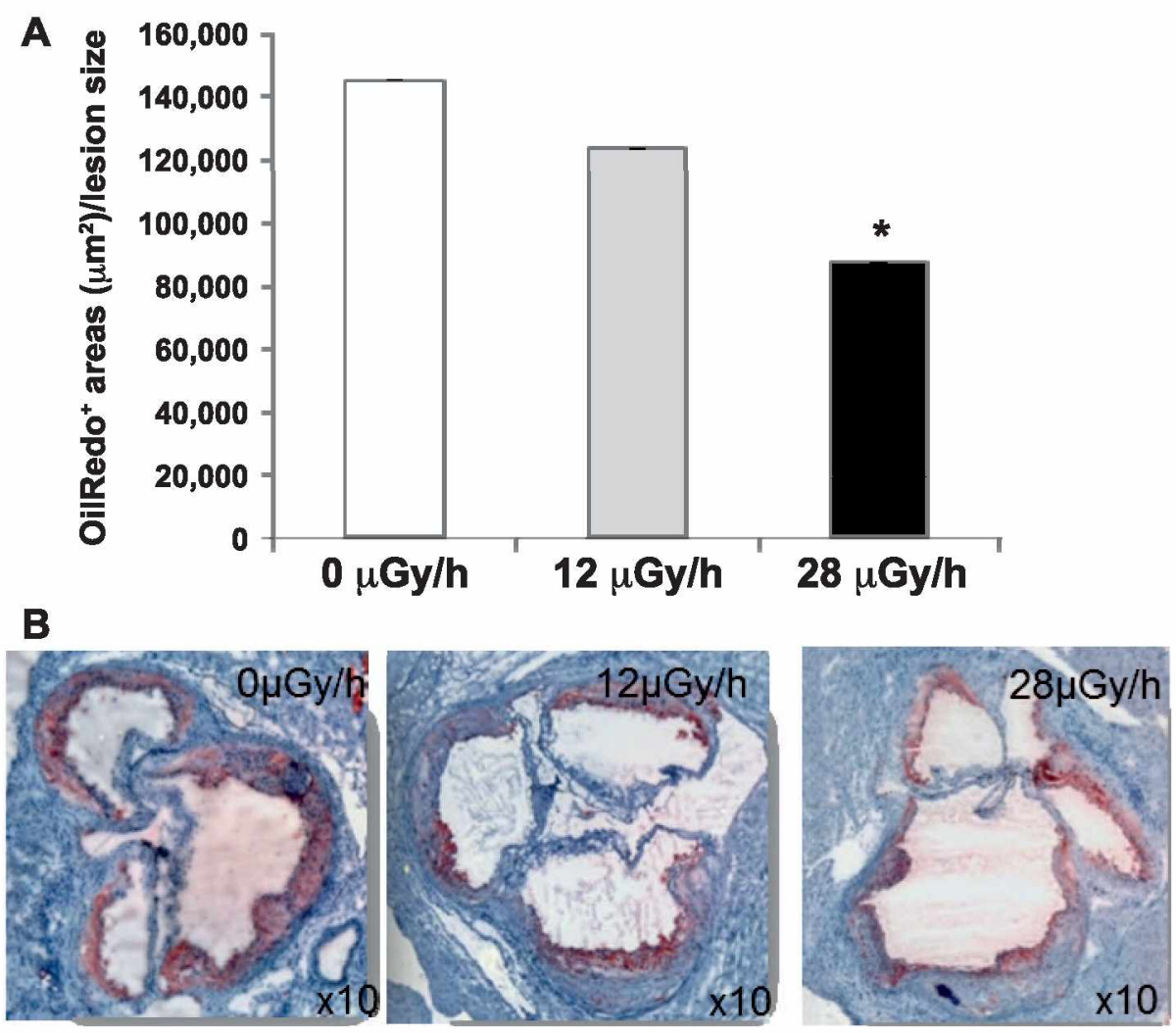

FIG. 4. Atheromatous lesion area is decreased after chronic gamma-ray irradiation for 8 months at $28 \mu \mathrm{Gy} / \mathrm{h}$. Panel A: Quantification of the average lesion area performed using Histolab software. Lesion areas are expressed as mean \pm SEM of $n=5$ to 7 sections per animal. Panel B: Representative pictures of oil red $O$ staining on aortic sinus cryosections after 8 months of radiation exposure (50× magnification).

of low-dose radiotherapy ( $<1$ Gy or fractionated) (39-41). Moreover, a previously published study highlighted that the anti-inflammatory effect of low-dose radiotherapy reduced adhesion of peripheral blood mononuclear cells to endothelium in vitro in the absence of effects on adhesion molecule expression (42).

However, in our current model, low-dose radiation does not decrease the expression of inflammatory parameters like VCAM-1, E selectin, IL6 or MCP1. Thus, the reduced levels of macrophages within the plaques of irradiated mice do not seem to result from a reduction in adhesion molecules, but could be partially explained by their migration function or polarity in atherosclerosis plaque.

In our study, reduced plaque macrophage content after low-dose exposure for 8 months suggests a potential plaque phenotype improvement. Indeed, in our study we observed a decrease in plaque size and an increase in collagen content, suggesting smaller and more stable plaque. The results on plaque stability are in accordance with chronic internal ${ }^{137} \mathrm{Cs}$ exposure found in another published study by our group (2I). By contrast, the decrease in plaque size is not observed after chronic internal ${ }^{137} \mathrm{Cs}$ exposure. Our study is in line with previously reported studies showing the beneficial effect of low-dose rate on atherosclerosis plaque development. On the contrary, the recently published study of Mancuso et al. has shown a detrimental effect on atherosclerosis development irrespective of dose rate; however, the cumulative low dose used in that study was higher $(0.3 \mathrm{~Gy})$ than the cumulated doses we used $(150$ $\mathrm{mGy}$ ). This result suggests that in the low-dose range, the effect on atherosclerosis is not linear with dose. Most clinical manifestations of atherosclerosis are related to plaque instability. Vulnerable human atherosclerotic plaque is characterized by increased accumulation of macrophages and decreased collagen content; this plaque is more prone to rupture than stable plaque (43-45). Schiller et al. demonstrated macrophage-rich and collagen-poor lesions in the aortic roots of irradiated $\mathrm{LDLR}^{-/-}$mice after acute high-dose (10 Gy) total-body irradiation. Another group (17) also observed an increase in macrophages in irradiated arteries. Differences in levels of inflammation may very well underlie the opposite outcomes of high- and low-dose radiation exposure.

In atherosclerosis progression, there is an imbalance of the cellular oxidants and anti-oxidant level with an increase in oxidant species generation. However, cells are equipped with an intricate cellular defense system that includes antioxidant enzymes (SOD, GSH, etc.) to scavenge oxidant species, thereby attenuating oxidant injury (46). We hypothesized that very low-dose radiation could stimulate anti-oxidant parameters and the anti- inflammatory profile of mice after low-dose and low-dose-rate irradiation. It was 

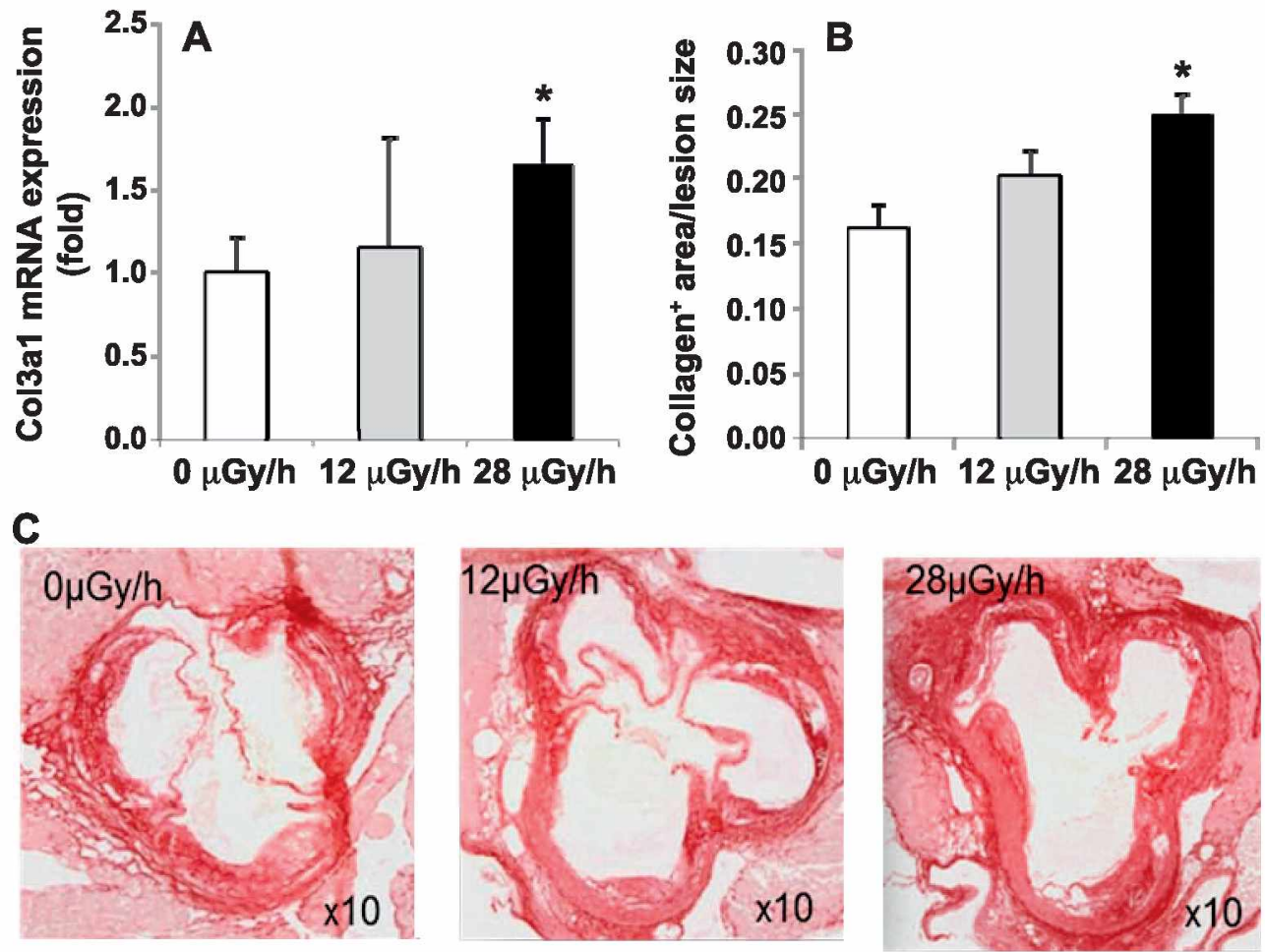

FIG. 5. Indices of atheromatous plaque stability are enhanced after chronic gamma-ray irradiation for 8 months at $28 \mu \mathrm{Gy} / \mathrm{h}$. Panel A: Quantification of collagen in the plaque shows that collagen was increased in the lesions. Panel B: Result shown in panel $\mathrm{A}$ was paralleled by an increase in col3 mRNA expression, assessed by RT-qPCR, in the entire aorta. Panel C: Picrosirius red staining for collagen was performed on aortic sinus cryosections. Representative images obtained at $100 \times$ magnification. $\mathrm{n}=12$ per animal. Results are expressed as mean \pm SEM of $n=12$ animals per group. $* P \leq 0.05$.

shown that low doses of $\mathrm{X}$ rays modulate the oxidative burst, which plays an anti-inflammatory role by reducing activated macrophages (47). These observations could be attributed to hormesis, whereby a damaging agent causing a mild stress response results in a beneficial effect. Whereas high-dose radiation increases oxidative stress and inflammation significantly, low-dose radiation modulates oxidative stress and inflammation $(48,49)$. However, in our experimental model we observed differences in oxidative stress parameters after low-dose irradiation. Specifically, we observed a significant increase in anti-oxidant parameters CAT, CTAT1, SOD1, SOD2 in low-dose irradiated animals compared to nonirradiated animals. Moreover, plaque ROS status, measured by DHE staining, was not altered by lowdose radiation. Thus, the increase in anti-oxidative parameters could regulate the level of oxidative stress. These results suggest that low-dose and low-dose-rate exposure may increase the mechanisms implicated in adaptive response, which counteract the initial stress and decrease inflammation and plaque phenotypes.

Our study demonstrates that chronic external low-dose exposure, comparable to what is measured in contaminated territories, does not potentiate atherosclerosis progression. On the contrary, we observed that such exposure, when it reached a cumulative dose of $150 \mathrm{mGy}$, enhanced a beneficial effect on atherosclerotic plaque in $\mathrm{ApoE}^{-1}$ mice, by increasing the expression of anti-inflammatory cytokines and anti-oxidative parameters, reducing plaque size and stimulating the accumulation of collagen. Our results related to nonlinearity are in agreement with some previously reported results $(19,21)$, while they are in contrast with those results of Mancuso et al. (31), in which deleterious effects of short-term exposure to a moderate radiation dose of 0.3 Gy were observed.

Further studies are needed to assess how early adaptive modifications in the plaques of mice exposed to low-dose ionizing radiation may alter lesion composition at later time points, with special consideration for mechanisms implicated in endothelial and smooth muscle cells.

\section{SUPPLEMENTARY INFORMATION}

Fig. S1. Aortic mRNA expression levels of pro- and antiinflammatory cytokines in C57B16 animals after 1.3 and $31.25 \mu \mathrm{Gy} / \mathrm{h}$ low-dose-rate irradiations for 8 months. Levels of CRP, IL4, IL13, IL18; TGF $\beta$, ICAM-1, IL6, MCP1 and TNF- $\alpha$ were determined using RT-PCR. GAPDH and HPRT were amplified and used as endogenous controls.

Fig. S2. Panel A: Representative images of superoxide production within the atheromatous plaques evaluated by DHE after 1 month exposure. No differences in DHE measurements were observed between groups. Panel B: 
Representative images of different time course of superoxide production within the atheromatous plaques evaluated by DHE. No differences in DHE measurements were observed between different conditions.

Fig. S3. Aortic mRNA enzyme expression levels in C57bl6 animals after chronic gamma irradiation for 8 months at $31.25 \mu \mathrm{Gy} / \mathrm{h}$. HO-1 and SOD-1 were significantly increased.

\section{ACKNOWLEDGMENTS}

We thank V. Joffres for secretarial assistance. This work was supported by Electricite de France (EDF).

Received: May 2, 2017; accepted: October 26, 2017; published online: December 11, 2017

\section{REFERENCES}

1. Takahashi I, Abbott RD, Ohshita T, Takahashi T, Ozasa K, Akahoshi $M$, et al. A prospective follow-up study of the association of radiation exposure with fatal and non-fatal stroke among atomic bomb survivors in Hiroshima and Nagasaki (19802003). BMJ Open 2012; 2:e000654.

2. Baker JE, Moulder JE, Hopewell JW. Radiation as a risk factor for cardiovascular disease. Antioxid Redox Signal 2011; 15:1945-56.

3. Galper SL, Yu JB, Mauch PM, Strasser JF, Silver B, Lacasce A, et al. Clinically significant cardiac disease in patients with Hodgkin lymphoma treated with mediastinal irradiation. Blood 2011; 117:412-8.

4. Martinou M, Gaya A. Cardiac complications after radical radiotherapy. Semin Oncol 2013; 40:178-85.

5. Jaworski C, Mariani JA, Wheeler G, Kaye DM. Cardiac complications of thoracic irradiation. JACC 2013; 61:2319-28.

6. Howe GR, Zablotska LB, Fix JJ, Egel J, Buchanan J. Analysis of the mortality experience amongst U.S. nuclear power industry workers after chronic low-dose exposure to ionizing radiation. Radiat Res 2004; 162:517-26.

7. Stewart JR, Fajardo LF. Radiation-induced heart disease. Clinical and experimental aspects. Radiol Clin North Am 1971; 9:511-31.

8. Stewart FA. Mechanisms and dose-response relationships for radiation-induced cardiovascular disease. Ann ICRP 2012; 41:72-9.

9. Stewart FA, Heeneman S, Te Poele J, Kruse J, Russell NS, Gijbels $\mathrm{M}$, et al. Ionizing radiation accelerates the development of atherosclerotic lesions in $\mathrm{ApoE}-/-$ mice and predisposes to an inflammatory plaque phenotype prone to hemorrhage. Am J Pathol 2006; 168:649-58.

10. Allahverdian S, Pannu PS, Francis GA. Contribution of monocytederived macrophages and smooth muscle cells to arterial foam cell formation. Cardiovasc Res 2012; 95:165-72.

11. Libby P. Changing concepts of atherogenesis. J Int Med 2000; 247:349-58.

12. Schultz-Hector S, Trott KR. Radiation-induced cardiovascular diseases: is the epidemiologic evidence compatible with the radiobiologic data? Int J Radiat Oncol Biol Phys 2007; 67:10-8.

13. Hendry JH, Akahoshi M, Wang LS, Lipshultz SE, Stewart FA, Trott KR. Radiation-induced cardiovascular injury. Radiat Environ Biophys 2008; 47:189-93.

14. Little MP, Tawn EJ, Tzoulaki I, Wakeford R, Hildebrandt G, Paris $\mathrm{F}$, et al. A systematic review of epidemiological associations between low and moderate doses of ionizing radiation and late cardiovascular effects, and their possible mechanisms. Radiat Res 2008; 169:99-109.

15. Bhatti P, Doody MM, Alexander BH, Yuenger J, Simon SL, Weinstock RM, et al. Breast cancer risk polymorphisms and interaction with ionizing radiation among U.S. radiologic technologists. Cancer Epidemiol Biomarkers Prev 2008; 17:2007-11.

16. Cottin Y, Kollum M, Kolodgie FD, Chan RC, Kim HS, Vodovotz $\mathrm{Y}$, et al. Intravascular radiation accelerates atherosclerotic lesion formation of hypercholesteremic rabbits. Cardiovasc Radiat Med $2001 ; 2: 231-40$.

17. Pakala R, Leborgne L, Cheneau E, Chan RC, Yazdi H, Fournadjiev J, et al. Radiation-induced atherosclerotic plaque progression in a hypercholesterolemic rabbit: a prospective vulnerable plaque model? Cardiovasc Radiat Med 2003; 4:146-51.

18. Hoving S, Heeneman S, Gijbels MJ, te Poele JA, Russell NS, Daemen MJ, et al. Single-dose and fractionated irradiation promote initiation and progression of atherosclerosis and induce an inflammatory plaque phenotype in $\mathrm{ApoE}(-/-)$ mice. Int J Radiat Oncol Biol Phys 2008; 71:848-57.

19. Mitchel RE, Hasu M, Bugden M, Wyatt H, Hildebrandt G, Chen $\mathrm{YX}$, et al. Low-dose radiation exposure and protection against atherosclerosis in ApoE(-/) mice: the influence of P53 heterozygosity. Radiat Res 2013; 179:190-9.

20. Baselet B, Rombouts C, Benotmane AM, Baatout S, Aerts A. Cardiovascular diseases related to ionizing radiation: The risk of low-dose exposure (Review). Int J Mol Med 2016; 38:1623-41.

21. Le Gallic C, Phalente Y, Manens L, Dublineau I, Benderitter M, Gueguen Y, et al. Chronic internal exposure to low dose $137 \mathrm{Cs}$ induces positive impact on the stability of atherosclerotic plaques by reducing inflammation in ApoE-/- mice. PloS One 2015; 10:e0128539.

22. Shao M, Lu X, Cong W, Xing X, Tan Y, Li Y, et al. Multiple lowdose radiation prevents type 2 diabetes-induced renal damage through attenuation of dyslipidemia and insulin resistance and subsequent renal inflammation and oxidative stress. PloS One 2014; 9:e92574.

23. Lodermann B, Wunderlich R, Frey S, Schorn C, Stangl S, Rodel F, et al. Low dose ionising radiation leads to a NF-kappaB dependent decreased secretion of active IL-1beta by activated macrophages with a discontinuous dose-dependency. Int J Radiat Biol 2012; 88:727-34.

24. Ebrahimian T, Le Gallic C, Stefani J, Dublineau I, Yentrapalli R, Harms-Ringdahl $\mathbf{M}$, et al. Chronic gamma-irradiation induces a dose-rate-dependent pro-inflammatory response and associated loss of function in human umbilical vein endothelial cells. Radiat Res 2015; 183:447-54.

25. Joven J, Rull A, Ferre N, Escola-Gil JC, Marsillach J, Coll B, et al. The results in rodent models of atherosclerosis are not interchangeable: the influence of diet and strain. Atherosclerosis 2007; 195:e85-92.

26. Livak KJ, Schmittgen TD. Analysis of relative gene expression data using real-time quantitative PCR and the 2(-delta delta $\mathrm{C}(\mathrm{T})$ ) method. Methods 2001; 25:402-8.

27. Daugherty A, Whitman SC. Quantification of atherosclerosis in mice. Methods Mol Biol 2003; 209:293-309.

28. Whitman SC, Ravisankar P, Elam H, Daugherty A. Exogenous interferon-gamma enhances atherosclerosis in apolipoprotein E-/mice. Am J Pathol 2000; 157:1819-24.

29. Gueguen Y, Lestaevel P, Grandcolas L, Baudelin C, Grison S, Jourdain JR, et al. Chronic contamination of rats with 137 cesium radionuclide: impact on the cardiovascular system. Cardiovasc Toxicol 2008; 8:33-40.

30. Bandazhevsky YI. Chronic Cs-137 incorporation in children's organs. Swiss Med Wkly 2003; 133:488-90.

31. Mancuso M, Pasquali E, Braga-Tanaka I, 3rd, Tanaka S, Pannicelli A, Giardullo P, et al. Acceleration of atherogenesis in ApoE-/mice exposed to acute or low-dose-rate ionizing radiation. Oncotarget $2015 ; 6: 31263-71$.

32. Cervelli T, Panetta D, Navarra T, Andreassi MG, Basta G, Galli A, et al. Effects of single and fractionated low-dose irradiation on vascular endothelial cells. Atherosclerosis $2014 ; 235: 510-8$. 
33. Wunderlich R, Ernst A, Rodel F, Fietkau R, Ott O, Lauber K, et al Low and moderate doses of ionizing radiation up to $2 \mathrm{~Gy}$ modulate transmigration and chemotaxis of activated macrophages, provoke an anti-inflammatory cytokine milieu, but do not impact upon viability and phagocytic function. Clin Exp Neuroimmunol 2015; 179:50-61.

34. Hallahan DE, Kuchibhotla J, Wyble C. Sialyl Lewis X mimetics attenuate E-selectin-mediated adhesion of leukocytes to irradiated human endothelial cells. Radiat Res 1997; 147:41-7.

35. Hildebrandt G, Loppnow G, Jahns J, Hindemith M, Anderegg U, Saalbach A, et al. Inhibition of the iNOS pathway in inflammatory macrophages by low-dose $\mathrm{X}$-irradiation in vitro. Is there a time dependence? Strahlenther Onkol 2003; 179:158-66.

36. Yentrapalli R, Azimzadeh O, Barjaktarovic Z, Sarioglu H, Wojcik A, Harms-Ringdahl $M$, et al. Quantitative proteomic analysis reveals induction of premature senescence in human umbilical vein endothelial cells exposed to chronic low-dose rate gamma radiation. Proteomics 2013; 13:1096-107.

37. Arenas M, Sabater S, Hernandez V, Rovirosa A, Lara PC, Biete A et al. Anti-inflammatory effects of low-dose radiotherapy. Indications, dose, and radiobiological mechanisms involved. Strahlenther Onkol 2012; 188:975-81.

38. Kataoka T. Study of antioxidative effects and anti-inflammatory effects in mice due to low-dose X-irradiation or radon inhalation. J Radiat Res 2013; 54:587-96.

39. von Pannewitz G. [Radiotherapy of arthrosis deformans. Method and results]. Der Radiologe 1970; 10:51-4.

40. Trott KR, Parker R, Seed MP. [The effect of $\mathrm{x}$-rays on experimental arthritis in the rat]. Strahlenther Onkol 1995; $171: 534-8$

41. Hildebrandt G, Radlingmayr A, Rosenthal S, Rothe R, Jahns J,
Hindemith M, et al. Low-dose radiotherapy (LD-RT) and the modulation of iNOS expression in adjuvant-induced arthritis in rats. Int J Radiat Biol 2003; 79:993-1001.

42. Kern PM, Keilholz L, Forster C, Hallmann R, Herrmann M, Seegenschmiedt MH. Low-dose radiotherapy selectively reduces adhesion of peripheral blood mononuclear cells to endothelium in vitro. Radiother Oncol 2000; 54:273-82.

43. Virmani R, Kolodgie FD, Burke AP, Farb A, Schwartz SM. Lessons from sudden coronary death: a comprehensive morphological classification scheme for atherosclerotic lesions. Arterioscler Thromb Vasc Biol 2000; 20:1262-75.

44. Aikawa M, Libby P. Lipid lowering therapy in atherosclerosis. Semin Vasc Med 2004; 4:357-66.

45. Virmani R, Kolodgie FD, Burke AP, Finn AV, Gold HK, Tulenko $\mathrm{TN}$, et al. Atherosclerotic plaque progression and vulnerability to rupture: angiogenesis as a source of intraplaque hemorrhage. Arterioscler Thromb Vasc Biol 2005; 25:2054-61.

46. Rodrigues SD, Franca KC, Dallin FT, Fujihara CK, Nascimento AJ, Pecoits-Filho R, et al. N-acetylcysteine as a potential strategy to attenuate the oxidative stress induced by uremic serum in the vascular system. Life Sci 2015; 121:110-6.

47. Schaue D, Marples B, Trott KR. The effects of low-dose Xirradiation on the oxidative burst in stimulated macrophages. Int $\mathrm{J}$ Radiat Biol 2002; 78:567-76.

48. Randolph GJ. Proliferating macrophages prevail in atherosclerosis. Nat Med 2013; 19:1094-5.

49. Robbins CS, Hilgendorf I, Weber GF, Theurl I, Iwamoto Y, Figueiredo JL, et al. Local proliferation dominates lesional macrophage accumulation in atherosclerosis. Nat Med 2013; 19:1166-72. 